\title{
Comparison by Pentafecta Criteria of Transperitoneal and Retroperitoneal Robotic Partial Nephrectomy for Large Renal Tumors
}

\author{
Chang II Choi, MD, ${ }^{1}$ Minyong Kang, MD, PhD, ${ }^{2}$ Hyun Hwan Sung, MD, PhD, ${ }^{2}$ Hwang Gyun Jeon, MD, PhD, \\ Byong Chang Jeong, $\mathrm{MD}, \mathrm{PhD}^{2}$, Seong Soo Jeon, $\mathrm{MD}, \mathrm{PhD}{ }^{2}$ \\ Hyun Moo Lee, MD, PhD, ${ }^{2}$ and Seong IL Seo, MD, $\mathrm{PhD}^{2}$
}

\begin{abstract}
Objective: To compare and analyze surgical and functional outcomes of transperitoneal robotic partial nephrectomy (TRPN) and retroperitoneal robotic partial nephrectomy (RRPN) in localized renal tumors, including $\geq 4 \mathrm{~cm}$ renal masses.

Methods: Of 566 consecutive patients who underwent robotic partial nephrectomy by a single surgeon from December 2008 through July 2017, records for 523 patients who were preoperatively and 1 year postoperatively evaluated were analyzed for estimated glomerular filtration rate (eGFR). Primary endpoint was a comparison of Pentafecta criteria (negative surgical margin, no 30-day complications, warm ischemic time [WIT] $\leq 25$ minutes, return of eGFR to $>90 \%$ from baseline, and no upstaging of chronic kidney disease) between TRPN and RRPN. Secondary endpoint was finding predictors for Pentafecta achievement.

Results: In all 523 patients, these Pentafecta criteria were lower for RRPN than TRPN: operation time $(p<0.001)$, WIT $(p=0.008)$, and estimated blood loss $(p=0.003)$. In patients with $\geq 4 \mathrm{~cm}$ renal tumors, only operation time was faster in RRPN than TRPN $(p=0.032)$. RRPN demonstrated more eGFR decrease in all patients $(p=0.006)$ and patients with $\geq 4 \mathrm{~cm}$ renal tumors $(p=0.008)$. Pentafecta achievements, complications, and recurrences were not significantly different between TRPN and RRPN in all patients and patients with $\geq 4 \mathrm{~cm}$ renal tumors. Multivariable analysis revealed baseline hemoglobin $(p=0.013)$ and tumor size $(p<0.001)$ were predictive for Pentafecta achievement.

Conclusions: Pentafecta achievement was similar for TRPN and RRPN. Baseline hemoglobin and tumor size were predictors of Pentafecta achievement. RRPN was properly performed for anterolateral renal tumor.
\end{abstract}

Keywords: large renal tumors, robotic partial nephrectomy, Pentafecta criteria

\section{Introduction}

$\mathbf{P}$ ARTIAL NEPHRECTOMY IS the standard treatment for localized T1 renal tumors when feasible and associated with lower incidence of chronic kidney disease (CKD) postoperatively than radical nephrectomy. ${ }^{1}$ Multiple studies report that laparoscopic partial nephrectomy (LPN) provides similar oncologic outcomes to radical nephrectomy and fewer complications, including perioperative blood loss, compared with open partial nephrectomy (OPN). No differences are seen in overall and progression-free survival between LPN and OPN. ${ }^{2-4}$ Nevertheless, LPN has a surgical learning curve that can extend ischemic time and delay renal reconstruction. Robotic partial nephrectomy (RPN) demon- strates better outcomes for warm ischemic time (WIT), estimated blood loss (EBL), and complication rates than LPN. ${ }^{5-9}$ Recent studies report RPN is safe for $\mathrm{cT} 1 \mathrm{~b}$ renal tumors and has similar oncological outcomes and complications to cT1a renal tumors. ${ }^{10-12}$ The feasibility of RPN in patients with cT2a renal tumors is reported to be the same as for patients with cT1 renal tumors, including complications, positive margins, hospital stays, and decline of renal function. ${ }^{13}$

Both transperitoneal and retroperitoneal approaches are used in minimally invasive partial nephrectomy. Approach is mainly determined by the location of the renal mass. The transperitoneal approach is recommended for anterior or lateral tumors and the retroperitoneal approach is recommended for posterior or posterolateral tumors. ${ }^{14}$

\footnotetext{
${ }^{1}$ Department of Urology, Hallym University Dongtan Sacred Heart Hospital, Hallym University School of Medicine, Hwaseong-si, Republic of Korea.

${ }^{2}$ Department of Urology, Samsung Medical Center, Sungkyunkwan University School of Medicine, Seoul, Republic of Korea.
} 
The retroperitoneal approach is performed in a confined space and therefore is more difficult, especially during LPN. In comparison, RPN has the advantages of a relatively transparent view and smaller devices by Endowrists on robotic instruments that stimulate and aid in the retroperitoneal approach.

By measuring perioperative and functional outcomes, the Pentafecta criteria could improve preservation of renal function after operation. ${ }^{15}$ Multiple studies compared Pentafecta outcomes after partial nephrectomy; however, only a single study compared transperitoneal robotic partial nephrectomy (TRPN) and retroperitoneal robotic partial nephrectomy (RRPN) using Pentafecta outcomes. ${ }^{16} \mathrm{We}$ found no comparative study using Pentafecta criteria on large renal tumors.

In this study, we compared perioperative and functional outcomes using Pentafecta criteria for all patients and we investigated prognostic factors for Pentafecta achievement. Furthermore, outcomes of TRPN and RRPN for large renal tumors of cT1b and cT2a tumors were compared by Pentafecta criteria to determine the feasibility of RPN.

\section{Methods}

\section{Patients and methods}

After obtaining Institutional Review Board approval, we performed a retrospective chart review of consecutive 567 patients who underwent RPN by a single experienced surgeon from December 2008 through July 2017 in Samsung Medical Center. RPN was performed for patients with localized renal tumors of $10 \mathrm{~cm}$ or less without locally advanced or metastatic disease. Patients with single kidney, bilateral renal tumors, multifocal tumors, and insufficient patient data were excluded.

We evaluated the following clinical and perioperative data: age at surgery, sex, body mass index (BMI), American Society of Anesthesiology (ASA) score, history of hypertension or diabetes, tumor size, tumor laterality, baseline hemoglobin, preoperative and postoperative estimated glomerular filtration rate (eGFR), clinical T stage, type of surgical approaches (transperitoneal or retroperitoneal), operation time, WIT, EBL, and length of stay (LOS). Operation time was defined as the time from the beginning (incision) to end of procedure (closure of the skin). Preoperative abdominopelvic computed tomography images were analyzed in the axial and coronal planes. R.E.N.A.L nephrometry score was assigned and categorized as low (4-6 points), intermediate (7-9 points), or high (10-12 points) complexity. Pathologic results were histology, Fuhrman grade, and pathologic $\mathrm{T}$ stage. Intraoperative and postoperative complications were graded by Clavien classification and recurrences were categorized into local recurrence and distant metastasis.

\section{Surgical approaches and technique}

Both transperitoneal and retroperitoneal approaches were performed. Decisions about approach were mainly based on tumor location. RRPN was performed for posterior or lateral tumors and TRPN for anterior or hilar tumors. Previous surgical history, complexity, perpendicular location of tumors, and large amounts of perirenal fat on preoperative images heavily influenced the choice of approach.
Intravenous mannitol was routinely infused before tumor resection. Tumors were identified by intraoperative ultrasonography and tumor margins were scored with electrocautery by monopolar scissors. All surgeries proceeded with warm ischemia by clamping the main renal arteries with laparoscopic bulldog clamps without using the selective-clamp techniques in both approaches. After resection, surgical beds were continuously sutured with 3-0 V-Loc absorbable sutures (Covidien). Using a sliding-clip technique, renal parenchyma was continuously sutured with 2-0 polyglactin (Covidien).

\section{Evaluation}

The primary endpoint was a comparison of TRPN and RRPN by Pentafecta criteria between all patients and patients with $\geq 4 \mathrm{~cm}$ renal tumors. Pentafecta criteria were first described by Zargar et al. and consist of negative cancer margin, WIT $\leq 25$ minutes, no complications, no CKD upstaging, and preservation of eGFR $>90 \%$ from baseline. ${ }^{15}$ Preservation of eGFR was defined as ratio of eGFR at 9 to 12 months after operation to preoperative eGFR. CKD stage was defined by 2012 Kidney Disease: Improving Global Outcomes (KDIGO) Clinical Practice Guideline for the Evaluation and Management of CKD. CKD upstaging included upstaging to stage III, IV, or V. ${ }^{17}$ As a secondary endpoint, we compared Pentafecta outcomes of both approaches in patients with $\geq 4 \mathrm{~cm}$ renal tumors. Subgroup analyses of anterior and posterior tumors were evaluated in the same manner. As a secondary endpoint, predictive factors for Pentafecta achievement were evaluated using multivariable logistic regression analysis.

\section{Statistics}

Chi-square tests, Student's $t$-tests, and Mann-Whitney $U$ tests were used for categorical and continuous variables. To compare preoperative and postoperative renal functional change, paired $t$-tests and Wilcoxon signed-rank tests were used. Multivariate cox regression analysis was used to find predictive factors for Pentafecta achievement. All tests were two sided, and $p$-values $<0.05$ were considered statistically significant. All statistical analyses were performed using SPSS Statistics 25.0 (IBM, Armonk, NY).

\section{Results}

Records for 523 patients undergoing TRPN $(n=310)$ and RRPN ( $n=213)$ with median follow-up of 35 months were analyzed. No significant differences were seen between TRPN and RRPN patients in age, BMI, history of hypertension or diabetes, laterality, ASA grade, clinical T stage, tumor size, and RENAL nephrometry score. Only sex $(p=0.023)$ and baseline hemoglobin were different $(p=0.004)$. After categorization by RENAL nephrometry score complexity, no differences were found between TRPN and RRPN for proportion of simple $(40.3 \%$ vs $43.7 \%)$, intermediate $(53.5 \%$ vs $50.2 \%)$, and complex (6.1\% vs $6.1 \%, p=0.731)$ scores. Analyses by separate RENAL nephrometry score domains revealed only domain A (anterior/posterior) was significantly different $(p=0.006$; " $\mathrm{A}$ " in Table 1). For pathological characteristics, tumor pathology and Fuhrman grade were not different; however, upstaging to pT3 stage was found for eight patients who received TRPN alone (2.6\%) ("B" in 
Table 1. Baseline Characteristics of All Patients

\begin{tabular}{|c|c|c|c|}
\hline $\begin{array}{l}\text { Variables } \\
\text { (A) Demographics and clinical characteristics }\end{array}$ & $\operatorname{TRPN}(\mathrm{n}=310)$ & $R R P N(\mathrm{n}=213)$ & $\mathrm{p}$-Value \\
\hline Age, years (median, IQR) & $51(42-58)$ & $50(41-58)$ & 0.595 \\
\hline $\begin{array}{l}\text { Sex, } n(\%) \\
\text { Male } \\
\text { Female }\end{array}$ & $\begin{array}{l}197(63.5) \\
112(36.5)\end{array}$ & $\begin{array}{r}156(73.2) \\
57(26.8)\end{array}$ & 0.023 \\
\hline $\begin{array}{l}\text { Hypertension, } n(\%) \\
\text { Diabetes, } n(\%) \\
\text { BMI, } \mathrm{kg} / \mathrm{m}^{2}(\text { median, IQR) } \\
\text { Baseline } \mathrm{Hb}, \mathrm{g} / \mathrm{dL} \text { (median, IQR) }\end{array}$ & $\begin{aligned} & 100(32.3) \\
& 34(11.0) \\
& 25.2(22.8-27.2) \\
& 14.4(13.3-15.2)\end{aligned}$ & $\begin{aligned} 63 & (29.6) \\
29 & (13.6) \\
25.0 & (22.8-27.0) \\
14.8 & (13.7-15.6)\end{aligned}$ & $\begin{array}{l}0.565 \\
0.412 \\
0.542 \\
0.004\end{array}$ \\
\hline $\begin{array}{l}\text { Laterality, } n(\%) \\
\text { Right } \\
\text { Left }\end{array}$ & $\begin{array}{l}152(49.0) \\
158(51.0)\end{array}$ & $\begin{array}{l}112(52.6) \\
101(47.4)\end{array}$ & 0.476 \\
\hline $\begin{array}{l}\text { ASA grade, } n(\%) \\
1 \\
2 \\
3\end{array}$ & $\begin{array}{l}135(43.5) \\
168(54.2) \\
\quad 7(2.3)\end{array}$ & $\begin{aligned} 90 & (42.3) \\
118 & (55.4) \\
5 & (2.3)\end{aligned}$ & 0.951 \\
\hline $\begin{array}{l}\text { Clinical T stage, } n(\%) \\
\text { cT1a } \\
\text { cT1b } \\
\text { cT2a }\end{array}$ & $\begin{aligned} 250 & (80.6) \\
57 & (18.4) \\
3 & (1.0)\end{aligned}$ & $\begin{aligned} 182 & (85.4) \\
29 & (13.6) \\
2 & (0.9)\end{aligned}$ & 0.341 \\
\hline $\begin{array}{l}\text { Tumor size, cm (median, IQR) } \\
\text { R.E.N.A.L score (median, IQR) } \\
\text { R.E.N.A.L score category }\end{array}$ & $\begin{aligned} 2.9 & (2.2-3.7) \\
7 & (6-8)\end{aligned}$ & $\begin{aligned} 2.8 & (2.0-3.5) \\
7 & (6-8)\end{aligned}$ & $\begin{array}{l}0.119 \\
0.506\end{array}$ \\
\hline $\begin{array}{l}\text { Low (4-6) } \\
\text { Intermediate }(7-9) \\
\text { High }(10-12)\end{array}$ & $\begin{array}{c}125(40.3) \\
166(53.5) \\
19(6.1)\end{array}$ & $\begin{aligned} & 93(43.7) \\
& 107(50.2) \\
& 13(6.1)\end{aligned}$ & 0.731 \\
\hline $\begin{array}{l}\text { R.E.N.A.L score domain " } \mathrm{N} \text { " } \\
1 \\
2 \\
3\end{array}$ & $\begin{array}{r}107(34.5) \\
95(30.6) \\
108(34.8)\end{array}$ & $\begin{array}{l}81(38.0) \\
67(31.5) \\
65(30.5)\end{array}$ & 0.551 \\
\hline $\begin{array}{l}\text { R.E.N.A.L score domain "A" } \\
\text { Anterior } \\
\text { Posterior } \\
\text { X }\end{array}$ & $\begin{array}{r}126(40.6) \\
85(27.4) \\
99(31.9)\end{array}$ & $\begin{array}{l}59(27.7) \\
79(37.1) \\
75(35.2)\end{array}$ & 0.006 \\
\hline (B) Histopathology & & & \\
\hline $\begin{array}{l}\text { Histology, } n(\%) \\
\text { Clear cell RCC } \\
\text { Papillary RCC } \\
\text { Chromophobe RCC } \\
\text { Other type RCC } \\
\text { Benign }\end{array}$ & $\begin{array}{r}237(76.5) \\
26(8.4) \\
20(6.5) \\
4(1.3) \\
23(7.4)\end{array}$ & $\begin{aligned} 174(81.7) \\
13(6.1) \\
14(6.6) \\
2(0.9) \\
10(4.7)\end{aligned}$ & 0.582 \\
\hline $\begin{array}{l}\text { Fuhrman grade, } n(\%) \\
\text { Low } \\
\text { High }\end{array}$ & $\begin{array}{l}151(52.6) \\
136(47.4)\end{array}$ & $\begin{array}{r}109(53.7) \\
94(46.3)\end{array}$ & 0.854 \\
\hline $\begin{array}{l}\text { Pathological T stage, } n(\%) \\
\text { pT1a } \\
\text { pT1b } \\
\text { pT2a } \\
\text { pT3 }\end{array}$ & $\begin{aligned} 255 & (82.3) \\
42 & (13.5) \\
5 & (1.6) \\
8 & (2.6)\end{aligned}$ & $\begin{array}{r}190(89.2) \\
22(10.3) \\
1(0.5) \\
0\end{array}$ & 0.025 \\
\hline
\end{tabular}

ASA = American Society of Anesthesiologists; $\mathrm{BMI}=$ body mass index; $\mathrm{Hb}=$ hemoglobin; $\mathrm{IQR}=$ interquartile range; $\mathrm{RCC}=$ renal cell carcinoma; RRPN = retroperitoneal robotic partial nephrectomy; TRPN = transperitoneal robotic partial nephrectomy.

Table 1). In these patients, six had cT1a stage, one had cT1b stage, and one had cT2a stage.

RRPN demonstrated lower operative time $(p<0.001)$, WIT $(p=0.008)$ and EBL $(p=0.003)$ than TRPN. Patients undergoing TRPN and RRPN had similar LOS, baseline eGFR, and postoperative 1-year eGFR. The rate of eGFR change at 1 year postoperation was significantly lower in
RRPN ( $p=0.006$; “A” in Table 2). Pentafecta achievement was similar for patients undergoing TRPN (34.5\%) and RRPN (40.8\%) $(p=0.167)$. Pentafecta components were analyzed separately and WIT $\leq 25$ minutes was the only discrete component (69.7\% TRPN vs $77.9 \%$ RRPN, $p=0.045)$. The rate of negative margins, absence of complications, eGFR recovery to $>90 \%$ of preoperative eGFR, 
Table 2. Comparison of Clinical Outcomes in All Patients

\begin{tabular}{|c|c|c|c|}
\hline $\begin{array}{l}\text { Outcomes } \\
\text { (A) Perioperative outcomes }\end{array}$ & $\operatorname{TRPN}(\mathrm{n}=310)$ & $R R P N(\mathrm{n}=213)$ & $\mathrm{p}$-Value \\
\hline Operation time, minutes (median, IQR) & $273(230-314)$ & $244(202-295)$ & $<0.001$ \\
\hline WIT, minutes (median, IQR) & $21(16-27)$ & $19(15-25)$ & 0.008 \\
\hline EBL, mL (median, IQR) & $150(100-200)$ & $100(60-200)$ & 0.003 \\
\hline LOS, days (median, IQR) & $9(8-9)$ & $9(8-9)$ & 0.891 \\
\hline Baseline eGFR, $\mathrm{mL} / \mathrm{min} / 1.73 \mathrm{~m}^{2}($ mean $\pm \mathrm{SD})$ & $86.1 \pm 17.7$ & $86.3 \pm 15.2$ & 0.779 \\
\hline Postoperative 1-year eGFR, mL/min/1.73 $\mathrm{m}^{2}($ mean $\pm \mathrm{SD})$ & $80.9 \pm 17.8$ & $78.4 \pm 15.1$ & 0.079 \\
\hline Changes of eGFR at 1 year postoperation, $\%$ (mean \pm SD) & $-5.4 \pm 14.3$ & $-8.7 \pm 12.4$ & 0.006 \\
\hline \multicolumn{4}{|l|}{ (B) Pentafecta outcomes } \\
\hline Pentafecta achievement, $n(\%)$ & $107(34.5)$ & $87(40.8)$ & 0.167 \\
\hline \multicolumn{4}{|l|}{ Pentafecta analysis, $n(\%)$} \\
\hline Negative margins & $306(98.7)$ & $213(100)$ & 0.150 \\
\hline WIT $\leq 25$ minutes & $216(69.7)$ & 166 (77.9) & 0.045 \\
\hline No complications & $265(85.5)$ & $172(80.8)$ & 0.186 \\
\hline eGFR $>90 \%$ of baseline & $183(59.0)$ & $121(56.8)$ & 0.652 \\
\hline No CKD upstaging & $274(88.4)$ & $194(91.1)$ & 0.385 \\
\hline
\end{tabular}

$\mathrm{CKD}=$ chronic kidney disease; $\mathrm{EBL}=$ estimated blood loss; eGFR = estimated glomerular filtration rate; LOS =length of stay; $\mathrm{SD}=$ standard deviation; WIT $=$ warm ischemic time.

and absence of CKD upstaging were comparable for the two approaches ("B" in Table 2).

Patients with $\geq 4 \mathrm{~cm}$ renal tumors were analyzed: $60 \mathrm{pa}-$ tients underwent TRPN and 31 underwent RRPN. No clinicopathological characteristics of patients with $\geq 4 \mathrm{~cm}$ renal tumors were different for the two approaches (Table 3). For patients with $\geq 4 \mathrm{~cm}$ renal tumors, operation time was shorter for RRPN ( $p=0.032)$, but WIT and EBL were not different. Baseline and 1 year postoperative eGFR were not significantly different between the two approaches. In patients with $\geq 4 \mathrm{~cm}$ renal tumors, changes in eGFR at 1 year postoperation were significantly lower at 1 year after RRPN ( $p=0.008$; " $A$ " in Table 4). Pentafecta achievement was not different $(p=0.428)$. More TRPN patients had eGFR $>90 \%$ of baseline than RRPN patients $(51.7 \%$ vs $29.0 \%, p=0.047)$. The rate of negative margins, WIT $\leq 25$ minutes, absence of complications, and absence of CKD upstaging were similar for both approaches ("B" in Table 4).

We performed subgroup analyses of patients with anterior and posterior renal tumors. Patients with anterior tumors had no differences in clinicopathological characteristics for the two approaches (Supplementary Table S1). EBL was significantly lower for RRPN ( $p=0.037$ ) and other outcomes, including Pentafecta achievement, were similar for both approaches (Supplementary Table S2). Patients with posterior tumors were similar for both approaches, except for the clinicopathological characteristic of laterality (Supplementary Table S3). Operation time was significantly shorter in RRPN ( $p<0.001)$ and other outcomes, including Pentafecta achievement, were not different (Supplementary Table S4).

Intraoperative and postoperative complications are in Table 5. No differences were observed for all patients or for patients with $\geq 4 \mathrm{~cm}$ renal tumors. A case of conversion to radical nephrectomy and a case of conversion to open procedure were found for T1a patients during TRPN. For all patients, no differences were found in major complications (Clavien grade $\geq 3$ ) between TRPN and RRPN. Neither blood transfusion $(p=0.629)$ nor postoperative ileus showed differences for the two approaches ( $p=0.274$; “ $\mathrm{A}$ " in Table 5).
For patients with $\geq 4 \mathrm{~cm}$ renal tumors, no injury to other organs and no conversion to radical nephrectomy or open procedure were found. Furthermore, no patients had major complications with either approach ("B" in Table 5).

For all patients, follow-up duration was longer in RRPN patients $(p=0.007)$. Recurrence was similar for both approaches $(p=0.830)$. Recurrences occurred only in TRPN patients as a single local recurrence and three distant metastases ("A" in Table 6). In patients with $\geq 4 \mathrm{~cm}$ renal tumors, median follow-up durations were longer in RRPN patients; however, differences were not significant because of limited cohort numbers $(p=0.202)$. Local recurrence and distant metastasis were single instances in TRPN patients ("B" in Table 6).

Multivariate logistic regression analysis was performed to find factors associated with Pentafecta achievement. We analyzed age, sex, history of hypertension or diabetes, BMI, baseline hemoglobin, tumor size, EBL, LOS, type of approach, tumor pathology, Fuhrman grade, and RENAL nephrometry score for domains E, N, A, and L. Multivariate analysis showed that baseline hemoglobin (odds ratio, OR [95\% confidence interval, 95\% CI] 1.182 [1.036-1.348], $p=0.013$ ) and tumor size (OR [95\% CI] 0.641 [0.536-0.767], $p<0.001)$ were associated with Pentafecta achievement. Type of approach (TRPN vs RRPN) $(p=0.141)$ had no correlation (Table 7).

\section{Discussion}

To our knowledge, four studies reported Pentafecta results after RPN. Our study is the largest RPN series using Pentafecta criteria as well as the largest comparison of TRPN and RRPN $(n=523)$. A single study compared TRPN and RRPN by Pentafecta criteria, but found variations in surgical procedures owing to multiple surgeons operating in two different centers. ${ }^{16}$

Trifecta criteria were generated by Hung and colleagues as WIT $\leq 25$ minutes, negative surgical margins, and no urological complications. ${ }^{18}$ Pentafecta criteria added $>90 \%$ preservation of baseline eGFR and no upgrading of CKD 
Table 3. Baseline Characteristics of Patients with $\geq 4$ cm Renal Tumors

\begin{tabular}{|c|c|c|c|}
\hline $\begin{array}{l}\text { Variables } \\
\text { (A) Demographics and clinical characteristics }\end{array}$ & $T R P N(\mathrm{n}=60)$ & $R R P N(\mathrm{n}=31)$ & $\mathrm{p}$-Value \\
\hline Age, years (median, IQR) & $50.0(40.5-57.0)$ & $53.0(39.5-60.0)$ & 0.752 \\
\hline $\begin{array}{l}\text { Sex, } n(\%) \\
\quad \text { Male } \\
\text { Female }\end{array}$ & $\begin{array}{l}35(58.3) \\
25(41.7)\end{array}$ & $\begin{array}{r}22(71.0) \\
9(29.0)\end{array}$ & 0.262 \\
\hline $\begin{array}{l}\text { Hypertension, } n(\%) \\
\text { Diabetes, } n(\%) \\
\text { BMI, kg/m }{ }^{2} \text { (median, IQR) } \\
\text { Baseline } \mathrm{Hb}, \mathrm{g} / \mathrm{dL} \text { (median, IQR) } \\
\text { Laterality, } n(\%)\end{array}$ & $\begin{aligned} 20 & (33.3) \\
8 & (13.3) \\
25.4 & (23.3-27.8) \\
14.1 & (13.0-15.2)\end{aligned}$ & $\begin{aligned} 10 & (32.3) \\
3 & (9.7) \\
24.7 & (22.6-28.5) \\
14.5 & (13.5-15.5)\end{aligned}$ & $\begin{array}{l}0.918 \\
0.743 \\
0.720 \\
0.429\end{array}$ \\
\hline $\begin{array}{l}\text { Right } \\
\text { Left }\end{array}$ & $\begin{array}{l}31(51.7) \\
29(48.3)\end{array}$ & $\begin{array}{l}15(48.4) \\
16(51.6)\end{array}$ & 0.827 \\
\hline $\begin{array}{l}\text { ASA grade, } n(\%) \\
1 \\
2\end{array}$ & $\begin{array}{l}25(41.7) \\
35(58.3)\end{array}$ & $\begin{array}{r}9(29.0) \\
22(71.0)\end{array}$ & 0.262 \\
\hline $\begin{array}{l}\text { Clinical T stage, } n(\%) \\
\text { cT1b } \\
\text { cT2a }\end{array}$ & $\begin{array}{c}57(95.0) \\
3(5.0)\end{array}$ & $\begin{aligned} 29 & (93.5) \\
2 & (6.5)\end{aligned}$ & 0.773 \\
\hline $\begin{array}{l}\text { Tumor size, cm (median, IQR) } \\
\text { R.E.N.A.L score (median, IQR) } \\
\text { R.E.N.A.L score category }\end{array}$ & $\begin{array}{l}4.7(4.3-5.4) \\
\quad 7(6-8)\end{array}$ & $\begin{array}{l}4.6(4.3-5.0) \\
\quad 7(6-8)\end{array}$ & $\begin{array}{l}0.537 \\
0.875\end{array}$ \\
\hline $\begin{array}{l}\text { K.E.N.A.L score category } \\
\text { Low (4-6) } \\
\text { Intermediate (7-9) } \\
\text { High }(10-12)\end{array}$ & $\begin{aligned} 18 & (30.0) \\
37 & (61.7) \\
5 & (8.3)\end{aligned}$ & $\begin{aligned} 10 & (32.3) \\
18 & (58.1) \\
3 & (9.7)\end{aligned}$ & 0.945 \\
\hline $\begin{array}{l}\text { R.E.N.A.L score domain " } N \text { " } \\
\quad 1 \\
2 \\
3\end{array}$ & $\begin{array}{l}10(16.7) \\
25(41.7) \\
25(41.7)\end{array}$ & $\begin{array}{r}6(19.4) \\
10(32.3) \\
15(48.4)\end{array}$ & 0.572 \\
\hline $\begin{array}{l}\text { R.E.N.A.L score domain "A" } \\
\text { Anterior } \\
\text { Posterior } \\
\text { X } \\
\end{array}$ & $\begin{array}{l}25(41.7) \\
20(33.3) \\
15(25.0)\end{array}$ & $\begin{array}{l}10(32.3) \\
11(35.5) \\
10(32.3)\end{array}$ & 0.641 \\
\hline (B) Histopathology & & & \\
\hline $\begin{array}{l}\text { Histology, } n(\%) \\
\text { Clear cell RCC } \\
\text { Papillary RCC } \\
\text { Chromophobe RCC } \\
\text { Other type RCC } \\
\text { Benign }\end{array}$ & $\begin{aligned} 44 & (73.3) \\
5 & (8.3) \\
6 & (10.0) \\
1 & (1.7) \\
4 & (6.7)\end{aligned}$ & $\begin{aligned} 22 & (71.0) \\
3 & (9.7) \\
4 & (12.9) \\
1 & (3.2) \\
1 & (3.2)\end{aligned}$ & 0.952 \\
\hline $\begin{array}{l}\text { Fuhrman grade, } n(\%) \\
\text { Low } \\
\text { High }\end{array}$ & $\begin{array}{l}23(41.1) \\
33(58.9)\end{array}$ & $\begin{array}{l}12(40.0) \\
18(60.0)\end{array}$ & 0.924 \\
\hline $\begin{array}{l}\text { Pathological T stage, } n(\%) \\
\text { pT1a } \\
\text { pT1b } \\
\text { pT2a } \\
\text { pT3 }\end{array}$ & $\begin{aligned} 16 & (26.7) \\
39 & (65.0) \\
3 & (5.0) \\
2 & (3.3)\end{aligned}$ & $\begin{array}{c}10(32.3) \\
20(64.5) \\
1(3.2) \\
0\end{array}$ & 0.705 \\
\hline
\end{tabular}

stage. Pentafecta achievement is considered composite measures of perioperative and functional outcomes. ${ }^{15}$ Zargar et al. analyzed 2392 cases of RPN and LPN in five highvolume centers by Pentafecta criteria; the rate of Pentafecta achievement for RPN was $38.5 \% .^{15}$ Our study found similar rates of Pentafecta achievement, with $34.5 \%$ for TRPN and $40.8 \%$ for RRPN for all patients. Kim and colleagues retrospectively analyzed 277 patients of renal cell carcinoma undergoing RPN and reported $38.3 \%$ of Pentafecta achievement for T1a patients and $26.7 \%$ for T1b patients. ${ }^{19}$ Our study also analyzed patients with $\geq 4 \mathrm{~cm}$ renal tumors. Rates of Pentafecta achievement for patients with $\geq 4 \mathrm{~cm}$ renal tumors decreased to $25.0 \%$ in patients receiving TRPN and $16.1 \%$ for patients receiving RRPN. To find the reason for decreased Pentafecta achievement in patients with $\geq 4 \mathrm{~cm}$ renal tumors, Pentafecta criteria were analyzed separately. We found that rates of WIT $\leq 25$ minutes were dissimilar for all patients and patients with $\geq 4 \mathrm{~cm}$ renal tumors. Moreover, in RRPN, preservation of eGFR $>90 \%$ was discordant for all patients and patients with $\geq 4 \mathrm{~cm}$ renal tumors $(65.8 \%$ for all 
Table 4. Comparison of Clinical Outcomes in Patients with $\geq 4$ cm Renal Tumors

\begin{tabular}{|c|c|c|c|}
\hline $\begin{array}{l}\text { Outcomes } \\
\text { (A) Perioperative outcomes }\end{array}$ & $T R P N(\mathrm{n}=60)$ & $R R P N(\mathrm{n}=31)$ & $\mathrm{p}-$ Value \\
\hline Operation time, minutes (median, IQR) & $310(272-353)$ & $269(227-330)$ & 0.032 \\
\hline WIT, minutes (median, IQR) & $26(21-30)$ & $24(21-29)$ & 0.572 \\
\hline EBL, mL (median, IQR) & $150(100-200)$ & $150(100-275)$ & 0.818 \\
\hline LOS, days (median, IQR) & $9(8-9)$ & $9(8-9)$ & 0.487 \\
\hline Baseline eGFR, $\mathrm{mL} / \mathrm{min} / 1.73 \mathrm{~m}^{2}($ mean $\pm \mathrm{SD})$ & $85.2 \pm 18.5$ & $87.7 \pm 17.7$ & 0.546 \\
\hline Postoperative 1 -year eGFR, $\mathrm{mL} / \mathrm{min} / 1.73 \mathrm{~m}^{2}($ mean $\pm \mathrm{SD})$ & $77.2 \pm 17.5$ & $72.5 \pm 14.2$ & 0.150 \\
\hline Changes of eGFR at 1 year postoperation, $\%$ (mean \pm SD) & $-8.3 \pm 14.2$ & $-16.5 \pm 12.7$ & 0.008 \\
\hline \multicolumn{4}{|l|}{ (B) Pentafecta outcomes } \\
\hline Pentafecta achievement, $n(\%)$ & $15(25.0)$ & $5(16.1)$ & 0.428 \\
\hline \multicolumn{4}{|l|}{ Pentafecta analysis, $n(\%)$} \\
\hline Negative margins & $59(98.3)$ & $31(100)$ & 0.999 \\
\hline WIT $\leq 25$ minutes & $29(48.3)$ & $17(54.8)$ & 0.659 \\
\hline No complications & $50(80.3)$ & $24(77.4)$ & 0.547 \\
\hline eGFR $>90 \%$ of baseline & $32(53.3)$ & $9(29.0)$ & 0.045 \\
\hline No CKD upstaging & $52(86.7)$ & $26(83.9)$ & 0.757 \\
\hline
\end{tabular}

and $29.0 \%$ for patients with $\geq 4 \mathrm{~cm}$ renal tumors). However, this disparity did not appear for TRPN patients (59.0\% for all and $51.7 \%$ for patients with $\geq 4 \mathrm{~cm}$ renal tumors). Therefore, TRPN could be more appropriate for achieving functional outcomes for patients with $\geq 4 \mathrm{~cm}$ renal tumors.

In secondary outcomes, baseline hemoglobin and tumor size were found to be independent predictive factors for Pentafecta achievement. Tumor size is a well-known factor that affects surgical difficulty. However, baseline hemoglobin is a new finding about which further studies are needed.

Our study demonstrated high negative margin rates, with $98.7 \%$ for TRPN and $100 \%$ for RRPN, which were superior to previous results from high-volume institutions. ${ }^{20,21} \mathrm{~A}$ recent study of RPN reported low positive surgical margin rates, with $3.3 \%$ for cT1b renal tumors and $4.5 \%$ for cT2a renal tumors. ${ }^{13}$ Our results with patients with $\geq 4 \mathrm{~cm}$ renal

Table 5. The Details of Intraoperative and Postoperative Complications

\begin{tabular}{|c|c|c|c|}
\hline $\begin{array}{l}\text { Complications } \\
\text { (A) All patients }\end{array}$ & $T R P N(\mathrm{n}=310)$ & $R R P N(\mathrm{n}=213)$ & p-Value \\
\hline Intraoperative, $n(\%)$ & $7(2.3)$ & $2(0.9)$ & \multirow[t]{7}{*}{0.322} \\
\hline Blood transfusion & $2(0.6)$ & $1(0.5)$ & \\
\hline Ureteral injury & 0 & $1(0.5)$ & \\
\hline Small bowel injury & $1(0.3)$ & 0 & \\
\hline Liver injury & $2(0.6)$ & 0 & \\
\hline Conversion to radical nephrectomy & $1(0.3)$ & 0 & \\
\hline Conversion to open procedure & $1(0.3)$ & 0 & \\
\hline Postoperative, $n(\%)$ & $41(13.2)$ & $39(18.3)$ & \multirow[t]{5}{*}{0.138} \\
\hline Clavien grade 1 & $26(8.4)$ & $29(13.6)$ & \\
\hline Clavien grade 2 & $13(4.2)$ & $7(3.3)$ & \\
\hline Clavien grade 3 & $2(0.6)$ & $3(1.4)$ & \\
\hline Clavien grade 4 & 0 & 0 & \\
\hline Blood transfusion, $n(\%)$ & $12(3.9)$ & $6(2.8)$ & 0.629 \\
\hline Ileus, $n(\%)$ & $3(1.0)$ & 0 & 0.274 \\
\hline $\begin{array}{l}\text { Complications } \\
\text { (B) Patients with } \geq 4 \mathrm{~cm} \text { renal tumors }\end{array}$ & Complications & $R R P N(\mathrm{n}=31)$ & $\mathrm{p}$-Value \\
\hline Intraoperative, $n(\%)$ & $1(1.7)$ & $1(3.2)$ & \multirow[t]{4}{*}{0.631} \\
\hline Blood transfusion & $1(1.7)$ & $1(3.2)$ & \\
\hline Injury of other organs & 0 & 0 & \\
\hline Surgical conversion & 0 & 0 & \\
\hline Postoperative, $n(\%)$ & $9(15.0)$ & $7(22.6)$ & \multirow[t]{4}{*}{0.683} \\
\hline Clavien grade 1 & $4(6.7)$ & $4(12.9)$ & \\
\hline Clavien grade 2 & $5(8.3)$ & $3(9.7)$ & \\
\hline Clavien grade $\geq 3$ & 0 & 0 & \\
\hline Blood transfusion, $n(\%)$ & $4(6.7)$ & $3(9.7)$ & 0.686 \\
\hline Ileus, $n(\%)$ & $2(3.3)$ & 0 & 0.546 \\
\hline
\end{tabular}


Table 6. Follow-Up Duration and Recurrence Rate

\begin{tabular}{lccr}
\hline (A) All patients & TRPN $(\mathrm{n}=310)$ & RRPN $(\mathrm{n}=213)$ & $\mathrm{p}$-Value \\
\hline $\begin{array}{l}\text { Follow up, months } \\
\text { Mean } \pm \text { SD }\end{array}$ & $34.2 \pm 25.6$ & $38.3 \pm 21.6$ & 0.007 \\
$\begin{array}{l}\text { Recurrences, } n(\%) \\
\quad \text { Local recurrence }\end{array}$ & $1(0.3)$ & 0 & 0.394 \\
$\quad$ Distant metastasis & $3(1.0)$ & 0 & $\mathrm{p}$-Value \\
\hline $\begin{array}{l}\text { (B) Patients with } \geq 4 \text { cm renal tumors } \\
\text { Follow up, months }\end{array}$ & $32.5 \pm 24.4$ & $39.2 \pm 21.7$ & 0.202 \\
$\quad$ Mean \pm SD & $1(1.7)$ & 0 & 0.590 \\
$\begin{array}{l}\text { Recurrences, } n(\%) \\
\text { Local recurrence }\end{array}$ & $1(1.7)$ & 0 & $\mathrm{n}=31)$ \\
$\quad$ Distant metastasis & & & \\
\hline
\end{tabular}

tumors also demonstrated lower positive margin rates with $1.7 \%$ for TRPN and $0 \%$ for RRPN. These results could be attributable to a single skillful surgeon performing surgeries in our study.

Our study demonstrated significantly shorter operation times for RRPN than TRPN for all patients $(p<0.001)$ and patients with $\geq 4 \mathrm{~cm}$ renal tumors ( $p=0.032$ ). These findings were comparable to results from prior studies and were because of the advantages of directly approaching the kidney without bowel mobilization in the retroperitoneal approach. ${ }^{16,22-25}$

RRPN was associated with benefits of shorter WIT $(p=0.008)$ and lower EBL $(p=0.003)$ in all patients, although no differences were seen in patients with $\geq 4 \mathrm{~cm}$ renal tumors. Shorter WIT and lower EBL could affect functional outcomes and are regarded as prominent factors in RPN. A single study described $307 \mathrm{~mL}$ less EBL for RRPN $(p<0.01)$, which widely uses earlier unclamping techniques in TRPN. ${ }^{24}$ The initial 107 RPN cases (57 TRPN and 50 RRPN) at our institution from 2008 to 2012 were reported by Choo and associates, with RRPN demonstrating shorter operative times $(p=0.015)$ and WIT $(p=0.04)$ than TRPN. However, EBL was not significantly different $(p=0.126) .{ }^{22}$
A meta-analysis reviewed eight studies on LPN and the retroperitoneal surgeries showed shorter LOS $(p<$ $0.001)$, shorter operative times $(p<0.001)$, and lower EBL $(p=0.042)$ than the transperitoneal surgeries. ${ }^{26}$ Nevertheless, retroperitoneal approach was not always associated with shorter LOS in RPN. Gin et al. and Kim et al. reported LOS benefits for RRPN. However, Stroup and colleagues reported no significant difference in LOS between the two approaches. ${ }^{16,27,28}$ Our study demonstrated no difference in LOS, possibly because of an absence of differences in perioperative complications, including ileus and blood transfusion. Also, RPN had the advantage of small Endowrists, which move easily when surgical space is limited, especially in retroperitoneal approach. They may contribute to less difference of complications and hospital stays between transperitoneal and retroperitoneal approaches in RPN.

Retrospective studies reported patients with cT2a tumors demonstrated less differences in eGFR at 12 months than patients with with cT1 tumors after open, LPN and RPN. ${ }^{13,29}$ Before our study, however, no studies have compared reduction in eGFR after one postoperative year between TRPN and RRPN in patients with $\geq 4 \mathrm{~cm}$ renal tumors. In our study,

Table 7. Multivariable Logistic Regression Analysis of Factors Predicting Pentafecta Achievement

\begin{tabular}{|c|c|c|c|c|c|c|}
\hline \multirow[b]{2}{*}{ Variables } & \multicolumn{3}{|c|}{ Univariable } & \multicolumn{3}{|c|}{ Multivariable } \\
\hline & $O R$ & $95 \% C I$ & $\mathrm{p}$-Value & $O R$ & $95 \% C I$ & $\mathrm{p}$-Value \\
\hline Age & 0.989 & $0.974-1.004$ & 0.145 & & & \\
\hline Sex (male $v s$ female) & 1.358 & $0.923-1.997$ & 0.12 & & & \\
\hline Hypertension & 0.779 & $0.528-1.149$ & 0.207 & & & \\
\hline Diabetes & 1.050 & $0.610-1.806$ & 0.861 & & & \\
\hline BMI & 1.015 & $0.963-1.069$ & 0.585 & & & \\
\hline Baseline $\mathrm{Hb}$ & 1.206 & $1.064-1.367$ & 0.003 & 1.182 & $1.036-1.348$ & 0.013 \\
\hline Tumor size & 0.603 & $0.505-0.720$ & $<0.001$ & 0.626 & $0.522-0.750$ & $<0.001$ \\
\hline EBL & 0.997 & $0.996-0.999$ & 0.003 & 0.998 & $0.997-1.000$ & 0.056 \\
\hline Types of approach (TRPN $v s$ RRPN) & 0.763 & $0.533-1.094$ & 0.141 & & & \\
\hline Tumor pathology (RCC vs benign) & 0.901 & $0.438-1.855$ & 0.778 & & & \\
\hline Fuhrman grade (low vs high) & 1.001 & $0.693-1.446$ & 0.994 & & & \\
\hline \multicolumn{7}{|l|}{ R.E.N.A.L score domain "N" } \\
\hline 1 & Reference & & & Reference & & \\
\hline 2 & 0.885 & $0.578-1.356$ & 0.575 & 1.139 & $0.724-1.792$ & 0.573 \\
\hline 3 & 0.507 & $0.327-0.788$ & 0.003 & 0.693 & $0.434-1.105$ & 0.123 \\
\hline
\end{tabular}

$\mathrm{CI}=$ confidence interval; $\mathrm{OR}=$ odds ratio. 
change in eGFR at 1 year postoperation was significantly more decreased at 1 year after RRPN than after TRPN for all patients $(p=0.006)$ and patients with $\geq 4 \mathrm{~cm}$ renal tumors ( $p=0.008$ ).In patients with $\geq 4 \mathrm{~cm}$ renal tumors, mean decrease in eGFR at 1 year postoperation was more than $10 \%$ for RRPN. In addition, TRPN demonstrated a higher rate of eGFR $>90 \%$ of baseline than RRPN (51.7\% vs $29.0 \%$, $p=0.047)$. Previous studies demonstrated that the transperitoneal approach had the advantage of sufficient surgical space as well as familiar anatomical sites. ${ }^{30-32}$ In comparison, confined surgical space created by a retroperitoneal approach potentially provoked difficulties in resecting large renal tumors with appropriate surgical margin lengths and suturing, which possibly injure the renal parenchyma. This may be attributable to the greater reduction of eGFR after RRPN.

In partial nephrectomy, a retroperitoneal approach has the advantage of avoiding the peritoneal cavity and having no impediment to the renal hilum, which results in less morbidity and faster recovery. ${ }^{33,34}$ Nevertheless, our study demonstrated no difference in perioperative complications, including transfusion and ileus, owing to the low incidence of complications of Clavien grade $\geq 2$, which was $4.8 \%$ for TRPN and $4.7 \%$ for RRPN.

We demonstrated notable findings in decision of approach. As in previous studies, TRPN was performed mainly for anterior tumors and RRPN for posterior tumors. Our study, however, determined that posterior tumors were $27.4 \%$ of TRPN cases and anterior tumors were $27.7 \%$ of RRPN cases. This prominence was a consequence of approach decisions by the surgeon, who preferred to operate on lateral tumors by RRPN and hilar tumors by TRPN. TRPN has been performed on posterior tumors with verified safety and effectiveness with no additional morbidity when comparing anterior or lateral tumors. ${ }^{35}$ Maurice and associates reported a matched analysis of TRPN and RRPN for posterior tumors with 370 cases. TRPN and RRPN achieved similar outcomes with a slight benefit in LOS and WIT for RRPN. ${ }^{36}$ We successfully operated on anterolateral tumors using RRPN and this might be attributable to the advantage of robotic surgery, including clear surgical view and small Endowrists.

This study has several limitations. The first is potential bias from the retrospective design and inclusion of only tertiary care patients in a single center. Second, renal scans were not examined for all patients. Therefore, differential renal functions could not be evaluated. Third, our median follow-up durations were 35 months and further long-term oncologic outcomes will be needed.

Despite these limitations, our study has the strength of including the largest number of Pentafecta studies after RPN and uniformity of a surgical procedure that has a learning curve, especially for RRPN. Our study supports a retroperitoneal approach for RPN, which demonstrated reliable functional outcomes for anterior as well as posterior renal tumors.

\section{Conclusion}

Pentafecta achievements were similar for TRPN and RRPN for all patients and patients with $\geq 4 \mathrm{~cm}$ renal tumors. Baseline hemoglobin and tumor size were predictive factors for Pentafecta achievement after RPN. In robotic surgery, a retroperitoneal approach could be suitable for anterolateral renal tumor.

\section{Author Disclosure Statement}

No competing financial interests exist.

\section{Funding Information}

This study was funded by a research grant from the National Research Foundation (NRF) of Korea, funded by the Ministry of Science and ICT (2017R1A2B4010568). This research was also supported by a grant from the Korea Health Technology R\&D Project through the Korea Health Industry Development Institute (KHIDI), funded by the Ministry of Health \& Welfare, Republic of Korea (HI17C0025).

\section{Supplementary Material}

Supplementary Table S1

Supplementary Table S2

Supplementary Table S3

Supplementary Table S4

\section{References}

1. Ljungberg B, Bensalah K, Canfield S, et al. EAU guidelines on renal cell carcinoma: 2014 update. Eur Urol 2015;67: 913-924.

2. Marszalek M, Meixl H, Polajnar M, Rauchenwald M, Jeschke K, Madersbacher S. Laparoscopic and open partial nephrectomy: A matched-pair comparison of 200 patients. Eur Urol 2009;55:1171-1178.

3. Gill IS, Kavoussi LR, Lane BR, et al. Comparison of 1,800 laparoscopic and open partial nephrectomies for single renal tumors. J Urol 2007;178:41-46.

4. Lane BR, Gill IS. 7-year oncological outcomes after laparoscopic and open partial nephrectomy. J Urol 2010;183: 473-479.

5. Patel HD, Mullins JK, Pierorazio PM, et al. Trends in renal surgery: Robotic technology is associated with increased use of partial nephrectomy. J Urol 2013;189:1229-1235.

6. Benway BM, Bhayani SB, Rogers CG, et al. Robot assisted partial nephrectomy versus laparoscopic partial nephrectomy for renal tumors: A multi-institutional analysis of perioperative outcomes. J Urol 2009;182:866-872.

7. Pierorazio PM, Patel HD, Feng T, Yohannan J, Hyams ES, Allaf ME. Robotic-assisted versus traditional laparoscopic partial nephrectomy: Comparison of outcomes and evaluation of learning curve. Urology 2011;78:813-819.

8. Khalifeh A, Autorino R, Hillyer SP, et al. Comparative outcomes and assessment of trifecta in 500 robotic and laparoscopic partial nephrectomy cases: A single surgeon experience. J Urol 2013;189:1236-1242.

9. Masson-Lecomte A, Bensalah K, Seringe E, et al. A prospective comparison of surgical and pathological outcomes obtained after robot-assisted or pure laparoscopic partial nephrectomy in moderate to complex renal tumours: Results from a French multicentre collaborative study. BJU Int 2013;111:256-263.

10. Luciani LG, Chiodini S, Mattevi D, et al. Robotic-assisted partial nephrectomy provides better operative outcomes as compared to the laparoscopic and open approaches: Results from a prospective cohort study. J Robot Surg 2017;11: 333-339.

11. Gettman MT, Blute ML, Chow GK, Neururer R, Bartsch G, Peschel R. Robotic-assisted laparoscopic partial nephrectomy: Technique and initial clinical experience with DaVinci robotic system. Urology 2004;64:914-918. 
12. Caruso RP, Phillips CK, Kau E, Taneja SS, Stifelman MD. Robot assisted laparoscopic partial nephrectomy: Initial experience. J Urol 2006;176:36-39.

13. Delto JC, Paulucci D, Helbig MW, et al. Robot-assisted partial nephrectomy for large renal masses: A multiinstitutional series. BJU Int 2018;121:908-915.

14. Ng CS, Gill IS, Ramani AP, et al. Transperitoneal versus retroperitoneal laparoscopic partial nephrectomy: Patient selection and perioperative outcomes. J Urol 2005;174:846-849.

15. Zargar H, Allaf ME, Bhayani S, et al. Trifecta and optimal perioperative outcomes of robotic and laparoscopic partial nephrectomy in surgical treatment of small renal masses: A multi-institutional study. BJU Int 2015;116:407-414.

16. Stroup SP, Hamilton ZA, Marshall MT, et al. Comparison of retroperitoneal and transperitoneal robotic partial nephrectomy for Pentafecta perioperative and renal functional outcomes. World J Urol 2017;35:1721-1728.

17. Levin A, Stevens PE. Summary of KDIGO 2012 CKD Guideline: Behind the scenes, need for guidance, and a framework for moving forward. Kidney Int 2014;85:49-61.

18. Hung AJ, Cai J, Simmons MN, Gill IS. "Trifecta" in partial nephrectomy. J Urol 2013;189:36-42.

19. Kim DK, Kim LH, Raheem AA, et al. Comparison of trifecta and pentafecta outcomes between $\mathrm{T} 1 \mathrm{a}$ and $\mathrm{T} 1 \mathrm{~b}$ renal masses following robot-assisted partial nephrectomy (RAPN) with minimum one year follow up: Can RAPN for T1b renal masses be feasible? PLoS One 2016;11:e0151738.

20. Bertolo RG, Zargar H, Autorino R, et al. Estimated glomerular filtration rate, renal scan and volumetric assessment of the kidney before and after partial nephrectomy: A review of the current literature. Minerva Urol Nefrol 2017; 69:539-547.

21. Minervini A, Campi R, Sessa F, et al. Positive surgical margins and local recurrence after simple enucleation and standard partial nephrectomy for malignant renal tumors: Systematic review of the literature and meta-analysis of prevalence. Minerva Urol Nefrol 2017;69:523-538.

22. Choo SH, Lee SY, Sung HH, et al. Transperitoneal versus retroperitoneal robotic partial nephrectomy: Matched-pair comparisons by nephrometry scores. World J Urol 2014;32: 1523-1529.

23. Pavan N, Derweesh I, Hampton LJ, et al. Retroperitoneal Robotic Partial Nephrectomy: Systematic Review and Cumulative Analysis of Comparative Outcomes. J Endourol 2018;32:591-596.

24. Hughes-Hallett A, Patki P, Patel N, Barber NJ, Sullivan M, Thilagarajah R. Robot-assisted partial nephrectomy: A comparison of the transperitoneal and retroperitoneal approaches. J Endourol 2013;27:869-874.

25. Tanaka K, Shigemura K, Furukawa J, et al. Comparison of the transperitoneal and retroperitoneal approach in robotassisted partial nephrectomy in an initial case series in Japan. J Endourol 2013;27:1384-1388.

26. Ren T, Liu Y, Zhao X, et al. Transperitoneal approach versus retroperitoneal approach: A meta-analysis of laparoscopic partial nephrectomy for renal cell carcinoma. PLoS One 2014;9:e91978.

27. Kim EH, Larson JA, Potretzke AM, Hulsey NK, Bhayani SB, Figenshau RS. Retroperitoneal robot-assisted partial nephrectomy for posterior renal masses is associated with earlier hospital discharge: A single-institution retrospective comparison. J Endourol 2015;29:1137-1142.

28. Gin GE, Maschino AC, Spaliviero M, Vertosick EA, Bernstein ML, Coleman JA. Comparison of perioperative outcomes of retroperitoneal and transperitoneal minimally invasive partial nephrectomy after adjusting for tumor complexity. Urology 2014;84:1355-1360.

29. Alanee S, Herberts M, Holland B, Dynda D. Contemporary experience with partial nephrectomy for stage T2 or greater renal tumors. Curr Urol Rep 2016;17:5.

30. Kieran K, Montgomery JS, Daignault S, Roberts WW, Wolf JS, Jr. Comparison of intraoperative parameters and perioperative complications of retroperitoneal and transperitoneal approaches to laparoscopic partial nephrectomy: Support for a retroperitoneal approach in selected patients. J Endourol 2007;21:754-759.

31. Wright JL, Porter JR. Laparoscopic partial nephrectomy: Comparison of transperitoneal and retroperitoneal approaches. J Urol 2005;174:841-845.

32. Marszalek M, Chromecki T, Al-Ali BM, et al. Laparoscopic partial nephrectomy: A matched-pair comparison of the transperitoneal versus the retroperitoneal approach. Urology 2011;77:109-113.

33. Nguyen MM, Gill IS. Halving ischemia time during laparoscopic partial nephrectomy. J Urol 2008;179:627-632; discussion 632.

34. Edge SB, Compton CC. The American Joint Committee on Cancer: The 7th edition of the AJCC cancer staging manual and the future of TNM. Ann Surg Oncol 2010;17:1471-1474.

35. Harris KT, Ball MW, Gorin MA, Curtiss KM, Pierorazio PM, Allaf ME. Transperitoneal robot-assisted partial nephrectomy: A comparison of posterior and anterior renal masses. J Endourol 2014;28:655-659.

36. Maurice MJ, Kaouk JH, Ramirez D, et al. Robotic partial nephrectomy for posterior tumors through a retroperitoneal approach offers decreased length of stay compared with the transperitoneal approach: A propensity-matched analysis. J Endourol 2017;31:158-162.

Address correspondence to: Seong IL Seo, MD, PhD Department of Urology Samsung Medical Center Sungkyunkwan University School of Medicine 81 Irwon-ro Gangnam-gu Seoul 06351 Republic of Korea

E-mail: siseo@skku.edu

$$
\begin{aligned}
& \text { Abbreviations Used } \\
\text { ASA } & =\text { American Society of Anesthesiology } \\
\text { BMI } & =\text { body mass index } \\
\mathrm{CI} & =\text { confidence interval } \\
\mathrm{CKD} & =\text { chronic kidney disease } \\
\mathrm{EBL} & =\text { estimated blood loss } \\
\mathrm{eGFR} & =\text { estimated glomerular filtration rate } \\
\mathrm{LOS} & =\text { length of stay } \\
\mathrm{LPN} & =\text { laparoscopic partial nephrectomy } \\
\mathrm{OPN} & =\text { open partial nephrectomy } \\
\mathrm{OR} & =\text { odds ratio } \\
\mathrm{RPN} & =\text { robotic partial nephrectomy } \\
\text { RRPN } & =\text { retroperitoneal robotic partial nephrectomy } \\
\text { TRPN } & =\text { transperitoneal robotic partial nephrectomy } \\
\mathrm{WIT} & =\text { warm ischemic time }
\end{aligned}
$$

